\title{
人参药材中人参皇苷的空间变异性及影响因子
}

\author{
郭 杰 ${ }^{1,2}$ 张 琴 ${ }^{1}$ 孙成忠 $^{3}$ 文 检 ${ }^{4}$ 谢彩香 $^{1^{*}}$ \\ ${ }^{1}$ 中国医学科学院/北京协和医学院药用植物研究所, 北京 $100193 ;{ }^{2}$ 武汉理工大学化学化工与生命科学学院, 武汉 $430070 ;{ }^{3}$ 中国测绘科学研究院, 北 \\ 京 $100830 ;{ }^{4}$ 成都中医药大学民族医药学院, 成都 611137
}

摘 要 为探究人参(Panax ginseng)㿝苷含量的空间变异性及其影响因子, 该研究以采自黑龙江、吉林、辽宁的 28 份六年生 人参为研究对象, 测定人参中 9 种单体㿝苷含量, 用单因素方差分析方法研究了人参㿝苷含量的空间变异性。并用主成分分 析、相关性分析和冗余分析法进行人参㿝苷含量与气候因子、土壤因子的相关性分析。结果表明: 吉林与辽宁人参的㿝苷含 量接近，且其含量高于黑龙江人参; 影响㿝苷含量的气候因子主要是降水，高温、日照时间是㿝苷含量的限制因子; 全氮、 铁、钾、有机质、 $\mathrm{pH}$ 值、锰、磷、锌对人参化学品质的影响较大。

关键词人参; 皇苷含量; 空间变异; 生态因子

引用格式: 郭杰, 张琴, 孙成忠, 文检, 谢彩香 (2017). 人参药材中人参㿝苷的空间变异性及影响因子. 植物生态学报, 41, 995-1002. doi: 10.17521/ cjpe.2016.0368

\section{Spatial variations of ginsenosides in Panax ginseng and their impact factors}

\author{
GUO Jie ${ }^{1,2}$, ZHANG Qin $^{1}$, SUN Cheng-Zhong ${ }^{3}$, WEN Jian ${ }^{4}$, and XIE Cai-Xiang ${ }^{1 *}$ \\ ${ }^{1}$ Institute of Medicinal Plant Development, Peking Union Medical College, Chinese Academy of Medical Sciences, Beijing 100193, China; ${ }^{2}$ School of Chemis- \\ try Engineering and Life Science, Wuhan University of Technology, Wuhan 430070, China; ${ }^{3}$ Chinese Academy of Surveying and Mapping, Beijing 100830, \\ China; and ${ }^{4}$ College of Ethnic Medicine, Chengdu University of Traditional Chinese Medicine, Chengdu 611137, China
}

\begin{abstract}
Aims This study aimed to reveal how ginsenosides content in Panax ginseng varied spatially and the regulating roles of environmental factors.

Methods Twenty eight P. ginseng samples were collected from Heilongjiang, Jilin and Liaoning provinces, and nine kinds of ginsenosides content in $P$. ginseng were measured. The one-way ANOVA was used to evaluate their spatial variations. The method of UPLC was employed to determine the content of nine kinds of ginsenosides in P. ginseng. The principal component analysis (PCA), correlation analysis (CA) and redundancy analysis (RDA) were used to analyze the relationship between ginsenosides content and ecological factors (including climate and soil factors).

Important findings The results showed that the content of ginsenosides in $P$. ginseng from Jilin and Liaoning was similar, and higher than that in Heilongjiang. Precipitation was the most important climate factor affecting the contents of ginsenosides. High temperature and strong sunshine limited the content of ginsenosides. The analysis on ginsenosides and soil factors indicated that soil nitrogen content, Fe, K, organic matter, $\mathrm{pH}$ value, $\mathrm{Mn}, \mathrm{P}, \mathrm{Zn}$ all had significant influences on the content of ginsenosides.
\end{abstract}

Key words Panax ginseng; ginsenosides content; spatial variation; ecological factor

Citation: Guo J, Zhang Q, Sun CZ, Wen J, Xie CX (2017). Spatial variations of ginsenosides in Panax ginseng and their impact factors. Chinese Journal of Plant Ecology, 41, 995-1002. doi: 10.17521/cjpe.2016.0368

影响药材品质的主要因素有遗传因素和环境 因素，两个因素共同作用决定药材品质的优劣(陈 士林等, 2007)。环境因素包括生态因素与生物因素, 生态因素是指环境中直接影响药材形态、生理和分 布的因素, 光照、温度、水分、空气、土壤是构成 生态因素的主要因子, 适宜的生长环境对优质药材
的形成起着至关重要的作用(陶曙红和吴凤锷, 2003; 唐仕欢等，2010；谢彩香等，2011)。Huang等(2010) 认为相对湿度和年降水量是影响黄花蒿(Artemisia annua)中青蒿素积累的主要影响因子, Dong等(2012) 认为土壤因子是影响枸杞(Lycium chinense)多酚类 成分含量的主要因素。Yang等(2011)发现玄参

收稿日期Received: 2016-12-02 接受日期Accepted: 2017-07-09

* 通信作者Author for correspondence (E-mail: caixiangxie@163.com) 
(Scrophularia ningpoensis)中玄参苷的积累与温度有 很强的负相关关系, 并且山䖿豆 (Lathyrus quinquenervius)的毒性成分 $\beta$-ODAP与土壤中 $\mathrm{K}^{+}$含量和 日照时间正相关，与土壤 $\mathrm{pH}$ 值负相关。阐明各生态 因子对药用植物品质的影响对于指导生产实践具有 重大意义。

人参药材为五加科植物人参 (Panax ginseng) 的 干燥根和根茎。具有大补元气, 补脾益肺, 生津养血, 安神益智的功效(张翠英等, 2010; 胥秀英等, 2011)。 我国人参的道地产区是辽宁、吉林、黑龙江三省。 人参皇苷是人参主要的活性成分, 也是评价人参品 质的标准(朱晶晶等, 2008; 林美妤等, 2011; 徐静等, 2011; 张翼输等, 2011)。人参㿝苷是人参在长期进 化中与环境相互作用的结果, 温度、日照时间、降 水等气候因子和土壤中营养元素含量等土壤因子直 接或间接影响其含量积累。研究道地药材的生态因 子和化学成分之间的相关性是道地药材品质评价的 重要思路(孟祥才等, 2010; 谢彩香等, 2011; 贾光林, 2012)。测定人参样品中人参㿝苷含量, 探讨不同产 地间人参样品㿝苷含量的差异, 再结合不同产地的 气候因子、土壤营养元素, 从而可找到造成人参皇 甘草量空间分布差异的主要因子。研究结果为濒危 物种人参的保护与利用、可持续发展及引种栽培提 供参考。

\section{1 材料和方法}

\section{1 材料}

人参药材的采收一般选生长 5 年以上的人参,
故本研究采用药材为黑龙江、吉林、辽宁 3 个产区 28 份六年生的人参栽培样品(表1), 采样原则依据每个 采样点按照对角交叉分别采集 5 株人参样品。人参样 品由中国医学科学院/北京协和医学院药用植物研 究所林余霖鉴定, 凭证标本保存于中国医学科学院 药用植物研究所标本馆。

\section{2 人参有效成分含量测定}

采用超高效液相色谱法测定人参中人参皇苷 $\mathrm{Rg} 1 、 \mathrm{Re} 、 \mathrm{Rb} 1 、 \mathrm{Rf} 、 \mathrm{Rg} 2 、 \mathrm{Rb} 2 、 \mathrm{Rb} 3 、 \mathrm{Rc}$ 和 $\mathrm{Rd} 9$ 种㿝苷的含量。色谱条件: Waters Acquity ${ }^{\mathrm{TM}}$ UPLC 超高效液相色谱仪, Waters中国有限公司, 色谱柱为 $\mathrm{BEH} \mathrm{C}_{18}(100 \mathrm{~mm} \times 2.1 \mathrm{~mm}, 1.7 \mu \mathrm{m})$, 沃特世(上海) 科技有限公司, 柱温: $35{ }^{\circ} \mathrm{C}$, 样品室温度 $4{ }^{\circ} \mathrm{C}$, 流 速: $0.4 \mathrm{~mL} \cdot \mathrm{min}^{-1}$, 检测波长: $203 \mathrm{~nm}$ 。以水(含 $10 \%$ 甲醇)为强洗，以水(含 $90 \%$ 甲醇)为弱洗，乙腈为流 动相 $\mathrm{A}$, 水为流动相 $\mathrm{B}$ 。采用梯度洗脱方法进行洗脱 (李慧等, 2015)。

\section{3 气候因子}

用数字高程模型(DEM)的多元线性回归插值方 法, 利用中国600多个气象站台30年(1980-2010)的 平均气候数据, 进行空间插值得到面域数据。气候 因子数据包括: 年平均湿度, 年活动积温, 年日照 时间, 年降水量, 年平均气温, 7 月平均气温, 1 月平 均气温。从气候数据网站(http://www.worldclim.org/) 获取月平均气候因子, 包括1-12月的月降水量和月 平均气温。

\section{4 人参产地土壤营养元素含量的测定}

采集 16 个人参栽培点的自然土壤(表2), 测定土

表1 人参样品来源

Table 1 Origins of Panax ginseng samples

\begin{tabular}{|c|c|c|c|}
\hline $\begin{array}{l}\text { 样品编号 } \\
\text { Sample code }\end{array}$ & 来源 Origin & $\begin{array}{l}\text { 样品编号 } \\
\text { Sample code }\end{array}$ & 来源 Origin \\
\hline H-NA & 黑龙江卧龙乡 Wolong, Heilongjiang & $\mathrm{J}-\mathrm{YH} 1$ & 吉林珲春市1 Huichun 1, Jilin \\
\hline $\mathrm{H}-\mathrm{HZ}$ & 黑龙江庄河村 Zhuanghe, Heilongjiang & $\mathrm{J}-\mathrm{YH} 2$ & 吉林珲春市2 Huichun 2, Jilin \\
\hline $\mathrm{H}-\mathrm{H}$ & 黑龙江省海伦市 Hailun, Heilongjiang & J-YH3 & 吉林珲春市3 Huichun 3, Jilin \\
\hline H-HB1 & 黑龙江北安县1 Bei'an 1, Heilongjiang & $\mathrm{J}-\mathrm{BC} 1$ & 吉林白山市1 Baishan 1, Jilin \\
\hline H-HB2 & 黑龙江北安县2 Bei'an 2, Heilongjiang & $\mathrm{J}-\mathrm{BC} 2$ & 吉林白山市2 Baishan 2, Jilin \\
\hline H-HX1 & 黑龙江逊克县1 Xunke 1, Heilongjiang & $\mathrm{J}-\mathrm{BX}$ & 吉林省新房子镇 Xinfangzi, Jilin \\
\hline H-HX2 & 黑龙江逊克县2 Xunke 2, Heilongjiang & J-JS & 吉林松江镇 Songjiang, Jilin \\
\hline $\mathrm{H}-\mathrm{HX} 3$ & 黑龙江逊克县3 Xunke 3, Heilongjiang & $\mathrm{J}-\mathrm{SL}$ & 吉林松原市 Songyuan, Jilin \\
\hline J-BFW & 吉林抚松县万良村 Wanliang, Fusong, Jilin & $\mathrm{J}-\mathrm{BJ}$ & 吉林靖宇县 Jingyu, Jilin \\
\hline $\mathrm{J}-\mathrm{BFX}$ & 吉林抚松县新屯子镇 Xintunzi, Fusong, Jilin & L-BH & 辽宁桓仁县 Huanren, Liaoning \\
\hline $\mathrm{J}-\mathrm{BFF}$ & 吉林抚松县枫林村 Fenglin, Fusong, Jilin & L-BHJ & 辽宁桓仁县巨户村 Juhu, Huanren, Liaoning \\
\hline $\mathrm{J}-\mathrm{BFD}$ & 吉林抚松县东岗镇 Donggang, Fusong, Jilin & L-DKX & 辽宁宽甸县下露河乡 Xialuhe, Kuandian, Liaoning \\
\hline $\mathrm{J}-\mathrm{TJ}$ & 吉林集安市 Ji’an, Jilin & L-DK & 辽宁宽甸县 Kuandian, Liaoning \\
\hline J-TJQ & 吉林集安市清河镇 Qinghe, Ji’an, Jilin & L-FQ & 辽宁抚顺市 Fushun, Liaoning \\
\hline
\end{tabular}

www.plant-ecology.com 
壤中 $\mathrm{Cu} 、 \mathrm{Fe} 、 \mathrm{Mn} 、 \mathrm{Zn}$ 、有机质、全氮、全磷、全 钾含量和 $\mathrm{pH}$ 值, 共 9 个土壤因子。应用二乙基三胺五 乙酸(DTPA)浸提-原子吸收分光光度法测定土壤中 $\mathrm{Cu} 、 \mathrm{Fe} 、 \mathrm{Mn} 、 \mathrm{Zn}$ 4种微量元素的含量。用电位法测 定测定土壤 $\mathrm{pH}$ 值。用铬酸氧还滴定法测定土壤有机 质含量。用半微量凯氏法测定土壤全氮含量。用酸 溶-钼锑抗比色法测定土壤全磷含量。用碱溶-火焰 光度法测定土壤全钾含量。

\section{5 分析方法}

应用SPSS 19.0进行单因素方差分析比较不同 产地间化学成分间的差异, 对不同产地气候因子进 行主成分分析, 利用相关性分析、咒余分析方法分 析人参皇苷含量和气候因子间的相关关系, 采用 Canoco 4.5 对土壤中营养元素与人参皇苷含量进行 圥余分析。

\section{2 结果}

对人参药材中人参皇苷的空间变异, 人参品质 与气候因子、土壤因子的相关性进行分析。

\section{1 不同产地人参皇苷含量分析}

为了比较不同地区人参品质是否存在差异性, 对不同产地人参样品中 9 种人参单体皇苷含量进行 单因素方差分析。假设各地人参品质无差异, 进行 单因素方差分析，该事件显著性水平越高，说明各 地人参品质越相似, 反之, 各产地间人参品质差异 性越大。

三个省份间两两比较结果(表3)显示, 黑龙江与 吉林间，除人参㿝苷 $R b 1 、 R g 2$ 外的 7 种人参㿝苷含 量存在的差异性最大; 黑龙江与辽宁间, 人参㿝苷 $\mathrm{Rb} 1 、 \mathrm{Rg} 2$ 的差异最大, 并且其他人参皇苷也具有较 大的差异(人参皇苷Re除外)。吉林与辽宁间，除人 参㿝苷 $\operatorname{Re}$ 外的 8 种人参㿝苷含量最接近, 并且其显 著性水平远大于黑龙江与吉林、辽宁间的比较结果。 因此可认为吉林与辽宁的人参品质比较接近, 各人 参㿝苷含量均无明显差异; 而黑龙江与吉林、辽宁 的人参品质有一定不同。

吉林与辽宁人参皇苷中除人参皇苷 $R b 3$ 的其余 8 种人参皇苷含量均大于黑龙江。《中华人民共和国

表2 采样点土壤样品

Table 2 Soil samples from collection sites of Panax ginseng

\begin{tabular}{ll|ll}
\hline $\begin{array}{l}\text { 样品编号 } \\
\text { Sample code }\end{array}$ & 来源 Origin & $\begin{array}{l}\text { 样品编号 } \\
\text { Sample code }\end{array}$ & 来源 Origin \\
\hline H-NA & 黑龙江卧龙乡Wolong, Heilongjiang & J-JS & 吉林松江镇 Songjiang, Jilin \\
H-HZ & 黑龙江庄河村 Zhuanghe, Heilongjiang & J-BC1 & 吉林白山市1 Baishan 1, Jilin \\
H-H & 黑龙江省海伦市 Hailun, Heilongjiang & J-BC2 & 吉林白山市2 Baishan 2, Jilin \\
H-HB1 & 黑龙江北安县1 Bei'an 1, Heilongjiang & J-YH3 & 吉林珲春市3 Huichun 3, Jilin \\
H-HB2 & 黑龙江北安县2 Bei'an 2, Heilongjiang & J-BX & 吉林省新房子镇 Xinfangzi, Jilin \\
J-BFX & 吉林抚松县新屯子镇 Xintunzi, Fusong, Jilin & J-BJ & 吉林靖宇县 Jingyu, Jilin \\
J-BFD & 吉林抚松县东岗镇 Donggang, Fusong, Jilin & L-DKX & 辽宁宽甸县下露河乡 Xialuhe Kuandian Liaoning \\
J-TJ & 吉林集安市 Ji’an, Jilin & L-DK & 辽宁宽甸县 Kuandian Liaoning \\
\hline
\end{tabular}

表3 人参单体㿝苷含量单因素方差分析

Table 3 One-way ANOVA of ginsenosides content in Panax ginseng

\begin{tabular}{|c|c|c|c|c|c|c|c|c|c|c|c|}
\hline \multirow{2}{*}{$\begin{array}{l}\text { 㿝苷 } \\
\text { Ginsenoside } \\
\operatorname{Rg} 1\end{array}$} & \multicolumn{2}{|c|}{$\begin{array}{l}\text { 人参产地 } \\
\text { P. ginseng origin }\end{array}$} & \multirow{2}{*}{$\begin{array}{l}\text { 显著性水平 } \\
\text { Significance level } \\
0.034\end{array}$} & \multirow{2}{*}{$\begin{array}{l}\text { 皇苷 } \\
\text { Ginsenoside } \\
\text { Rf }\end{array}$} & \multicolumn{2}{|c|}{$\begin{array}{l}\text { 人参产地 } \\
P . \text { ginseng origin }\end{array}$} & \multirow{2}{*}{ 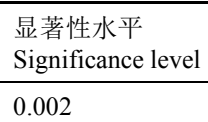 } & \multirow{2}{*}{\begin{tabular}{|l}
$\begin{array}{l}\text { 㿝苷 } \\
\text { Ginsenoside }\end{array}$ \\
Rb2
\end{tabular}} & \multicolumn{2}{|c|}{$\begin{array}{l}\text { 人参产地 } \\
\text { P. ginseng origin }\end{array}$} & \multirow{2}{*}{ 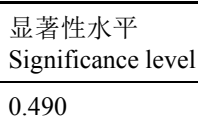 } \\
\hline & $\mathrm{H}$ & $\mathrm{J}$ & & & $\mathrm{H}$ & $\mathrm{J}$ & & & $\mathrm{H}$ & $\mathrm{J}$ & \\
\hline & & $\mathrm{L}$ & 0.279 & & & $\mathrm{~L}$ & 0.072 & & & $\mathrm{~L}$ & 0.621 \\
\hline & $\mathrm{J}$ & $\mathrm{L}$ & 0.506 & & $\mathrm{~J}$ & $\mathrm{~L}$ & 0.403 & & $\mathrm{~J}$ & $\mathrm{~L}$ & 0.967 \\
\hline \multirow[t]{3}{*}{$\mathrm{Re}$} & $\mathrm{H}$ & J & 0.300 & $\operatorname{Rg} 2$ & $\mathrm{H}$ & $\mathrm{J}$ & 0.248 & $\mathrm{Rb} 3$ & $\mathrm{H}$ & $\mathrm{J}$ & 0.439 \\
\hline & & $\mathrm{L}$ & 0.737 & & & $\mathrm{~L}$ & 0.194 & & & $\mathrm{~L}$ & 0.770 \\
\hline & $\mathrm{J}$ & $\mathrm{L}$ & 0.606 & & $\mathrm{~J}$ & $\mathrm{~L}$ & 0.641 & & $\mathrm{~J}$ & $\mathrm{~L}$ & 0.736 \\
\hline \multirow[t]{3}{*}{$\mathrm{Rb} 1$} & $\mathrm{H}$ & $\mathrm{J}$ & 0.086 & Rc & $\mathrm{H}$ & $\mathrm{J}$ & 0.267 & $\mathrm{Rd}$ & $\mathrm{H}$ & $\mathrm{J}$ & 0.053 \\
\hline & & $\mathrm{L}$ & 0.053 & & & $\mathrm{~L}$ & 0.272 & & & $\mathrm{~L}$ & 0.134 \\
\hline & $\mathrm{J}$ & $\mathrm{L}$ & 0.475 & & $\mathrm{~J}$ & $\mathrm{~L}$ & 0.783 & & $\mathrm{~J}$ & $\mathrm{~L}$ & 0.988 \\
\hline
\end{tabular}

$\mathrm{H}$, 黑龙江; J, 吉林; L, 辽宁。

H, Heilongjiang; J, Jilin; L, Liaoning. 
药典》 (2015版)中以人参㿝苷 $\operatorname{Rg}_{1}$ 和人参㿝苷 $R e$ 的总 量、人参皇苷 $\mathrm{Rb}_{1}$ 的含量为指标评价人参质量。本研 究中, 人参皇苷 $\operatorname{Rg} 1$ 和 $R e$ 总量各地平均含量吉林 (3.693 $\left.\mathrm{mg} \cdot \mathrm{g}^{-1}\right)>$ 辽宁 $\left(3.095 \mathrm{mg} \cdot \mathrm{g}^{-1}\right)>$ 黑龙江 $(2.618$ $\left.\mathrm{mg} \cdot \mathrm{g}^{-1}\right)$, 人参皇苷 $\mathrm{Rb} 1$ 各地平均含量辽宁 $(1.453$ $\left.\mathrm{mg} \cdot \mathrm{g}^{-1}\right)>$ 吉林 $\left(1.227 \mathrm{mg} \cdot \mathrm{g}^{-1}\right)>$ 黑 龙江 $(0.756$ $\left.\mathrm{mg} \cdot \mathrm{g}^{-1}\right)$ 。结果显示吉林、辽宁人参皇苷含量高于黑 龙江。

上述分析结果显示, 人参㿝苷含量呈现出空间 变异现象。通过主成分分析篮选出对人参皇苷含量 空间变异影响较大的单体㿝苷, 图1中显示提取的 3 个主成分，其方差贡献率分别为 $52.983 \% 、 17.376 \%$ 、 $10.796 \%$, 累计方差贡献率大于 $80 \%$, 主成分一主要 由人参皇苷Rc、Rb2、Rb1、Rf组成, 主成分二载荷 较大的因子是人参皇苷 $R g 1$, 主成分三的主要因子 为人参皇苷 $R e$ 。各样品中, $R g 1 、 R e 、 R b 1 、 R f 、 R c 、$ $\mathrm{Rb} 26$ 种人参皇苷含量较大, 对于大多数人参样品, 这6种人参㿝苷含量总和达到皇苷总量的 $90 \%$ 以上, 最小的也有 $87 \%$ 。所以可认为 $R g 1 、 R e 、 R b 1 、 R f 、$ $R c 、 R b 2$ 6种人参皇苷是造成人参皇苷含量空间变 异的主要化学成分。

\section{2 人参产地气候因子分析}

对人参样品点的气候因子进行主成分分析 (PCA), 选取气候因子包括年平均湿度、年活动积 温、年日照时间、年降水量、年平均气温、7月平均 气温、1月平均气温、6月降水量、7月降水量、8月 降水量等。分析结果(图2)显示, 黑龙江采样点主要

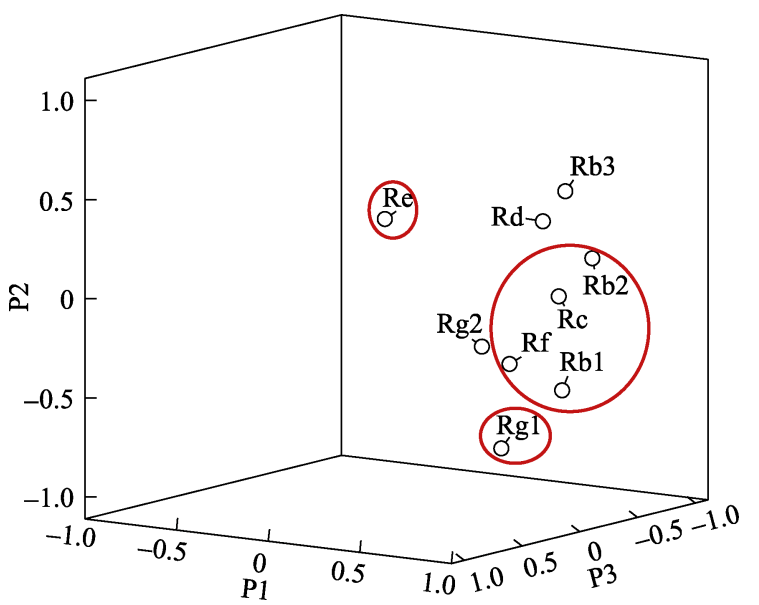

图1 人参皇苷 $(\mathrm{Rg} 1 、 \mathrm{Re} 、 \mathrm{Rb} 1 、 \mathrm{Rf} 、 \mathrm{Rg} 2 、 \mathrm{Rb} 2 、 \mathrm{Rb} 3 、 \mathrm{Rc}$ 和Rd)主成分分析载荷图。

Fig. 1 Principal component analysis loading plot of ginsenosides (Rg1, Re, Rb1, Rf, Rg2, Rb2, Rb3, Rc and Rd) in Panax ginseng.

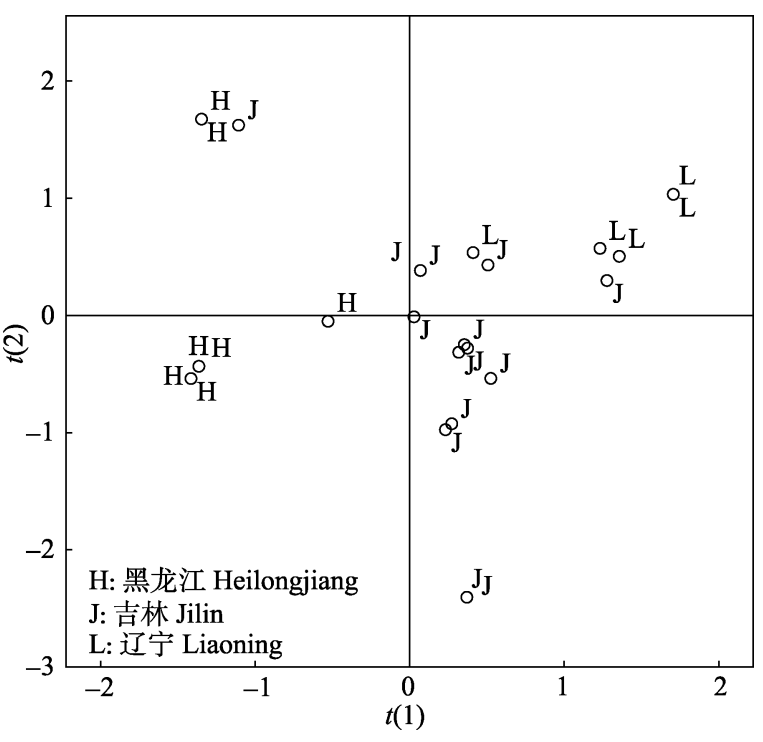

图2 人参产地气候因子主成分分析。

Fig. 2 Principal component analysis scatter plot of climate factors in Panax ginseng regions.

分布在坐标轴左侧; 吉林、辽宁采样点主要分布在 坐标轴右侧, 且吉林与辽宁采样点比较接近, 说明 辽宁、吉林的气候比较接近, 而与黑龙江的气候有 一定差异。这与上文中对人参皇苷含量的单因素方 差分析的分析结果相一致。

人参样品点的年平均生态因子 PCA载荷量表明, 第一主成分方差贡献率为 $45.75 \%$, 且载荷较大的因 子有年降水量、 8 月降水量, 主要反映了采样点的降 水条件, 可认为是影响人参㿝苷积累的水分因子。 第二主成分方差贡献率为 $32.21 \%$, 载荷较大的因子 有7月平均气温、年活动积温, 可认为是影响人参皇 苷积累的热量条件; 第三主成分方差贡献率为 $14.47 \%$, 载荷较大的因子为年日照时间, 是影响人 参皇苷积累的光照因子。水分因子、热量因子与光 照因子累计方差贡献率达到 $92.43 \%$, 所以, 影响人 参㿝苷积累的主要气候因子是水分、热量和光照, 并且水分因子对人参㿝苷积累影响最大。

\section{3 气候因子与皇苷含量相关性分析}

为了进一步分析人参皇苷含量对各气候因子 的响应情况, 对气候因子与主要人参皇苷 Rg $1 、 R e 、$ $R b 1 、 R f 、 R c 、 R b 2$ 的含量进行相关性分析。

相关性分析结果(表4)显示, 年平均湿度、年降 水量与人参皇苷含量均为正相关, 6 月降水量与人参 㿝苷含量均为正相关，7月降水量与除人参皇苷 $\mathrm{Rg} 1 、 \mathrm{Rb} 2$ 外的其余 4 种人参皇苷含量均为正相关, 8 月平均气温与人参皇苷 $\mathrm{Rb} 1 、 \mathrm{Rf} 、 \mathrm{Rc}$ 含量为正相关, 
表4 人参皇苷含量与气候因子相关性分析

Table 4 Correlation analysis of climate factors and ginsenosides content in Panax ginseng

\begin{tabular}{lrrrrrr}
\hline 气候因子 Climate factor & \multicolumn{5}{c}{ 人参㿝苷 Ginsenoside } \\
\cline { 2 - 7 } & \multicolumn{1}{c}{$\mathrm{Rg} 1$} & $\mathrm{Re}$ & $\mathrm{Rb} 1$ & $\mathrm{Rf}$ & $\mathrm{Rc}$ & $\mathrm{Rb} 2$ \\
\hline 年平均湿度 Annual average humidity & 0.156 & 0.249 & 0.104 & 0.224 & 0.118 & 0.044 \\
年活动积温 Annual active accumulated temperature & -0.337 & -0.212 & -0.171 & -0.210 & -0.037 & -0.122 \\
年日照时间 Annual sunshine & -0.190 & -0.102 & -0.149 & -0.322 & -0.026 & 0.063 \\
年降水量 Annual precipitation & 0.296 & 0.181 & 0.306 & 0.502 & 0.108 & 0.010 \\
年平均气温 Average Annual temperature & 0.043 & 0.027 & 0.142 & 0.210 & 0.030 & 0.121 \\
7月平均气温 Average temperature in July & -0.358 & -0.167 & -0.217 & -0.303 & -0.095 & -0.167 \\
1月平均气温 Average temperature in January & 0.260 & 0.008 & 0.211 & 0.297 & -0.010 & -0.127 \\
6月降水量 Precipitation in June & 0.189 & 0.251 & 0.246 & 0.504 & 0.218 & 0.081 \\
7月降水量 Precipitation in July & -0.059 & 0.045 & 0.103 & 0.205 & 0.065 & -0.042 \\
8月降水量 Precipitation in August & -0.029 & -0.011 & 0.101 & 0.177 & 0.009 & -0.125 \\
\hline
\end{tabular}

说明降水量较大的潮湿环境更为适合人参皇苷的积 累，但是在人参生长过程中也应合理控制水分; 年 平均气温与人参㿝苷含量均为正相关, 1 月平均气温 与除人参皇苷 $R c 、 R b 2$ 外的其余 4 种人参皇苷含量均 为正相关，年活动积温、7月平均气温与各人参皇苷 含量均为负相关, 说明人参喜温暖的气候条件, 但 高温会抑制人参皇苷的积累; 年日照时间与各人参 㿝苷含量均为负相关, 说明阴凉的环境对人参皇苷 的积累较为有利。总的来说, 人参喜潮湿、阴凉的 生态环境，高温与强光照均会抑制各人参皇苷的积

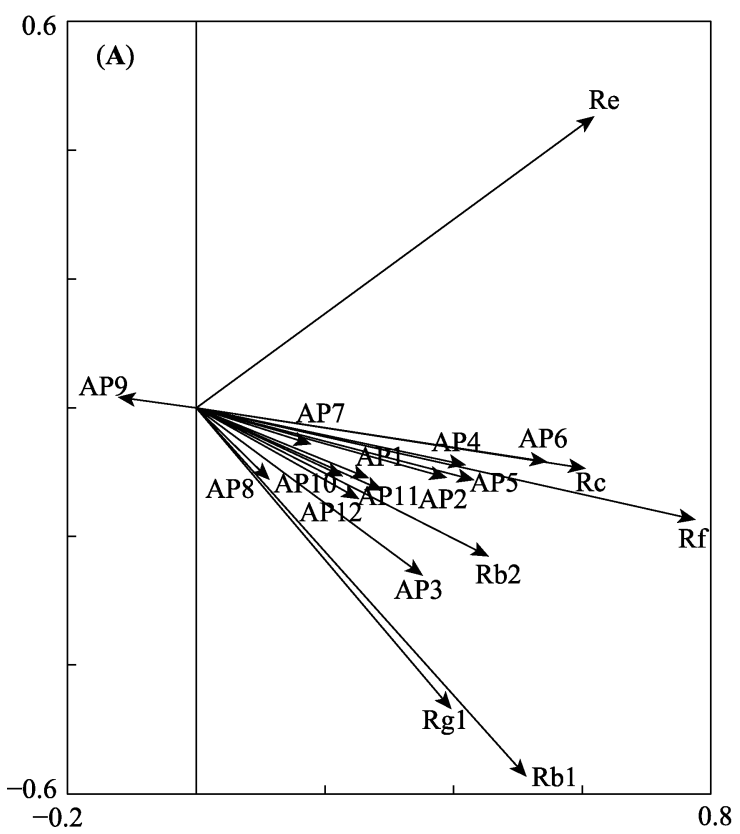

累，从而对人参品质产生影响。

为进一步分析热量和水分因子对人参品质的 影响，对不同采样点的月降水量和月平均气温进行 圥余分析。

根据图 $3 \mathrm{~A}$ ，除 9 月外的其余 11 个月降水量均与 人参皇苷含量呈正相关关系，且 9 月降水量对人参 㿝苷含量影响较小，说明较大的降水量有利于人参 㿝苷的积累，这与上文中降水量与人参皇苷含量正 相关的分析结果一致。图3B显示, 6-9月的月平均气 温与人参皇苷含量负相关, 其余月份月平均气温与

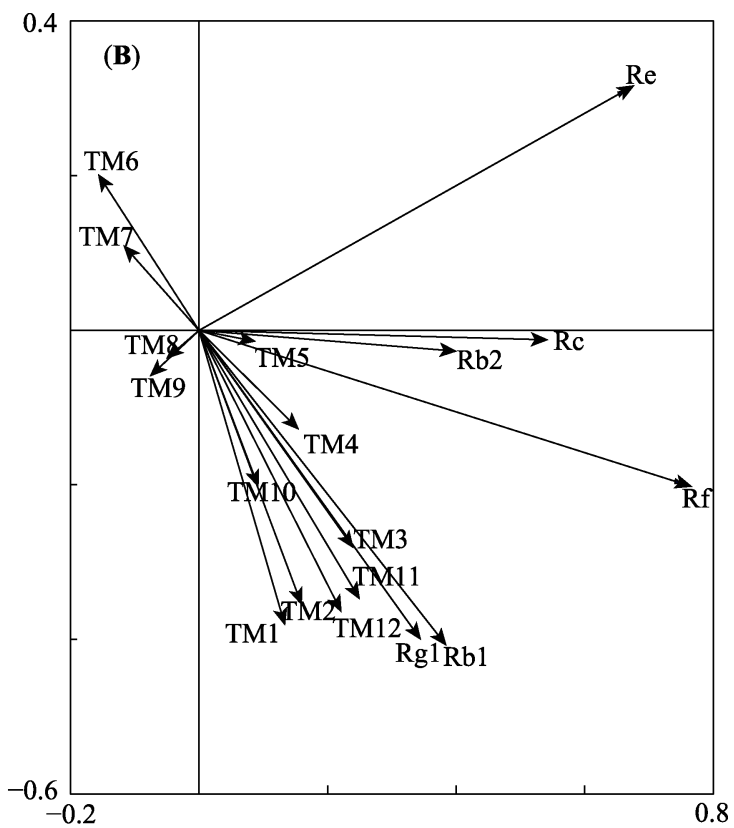

图3 人参皇苷 $(\mathrm{Rb} 1 、 \mathrm{Rb} 2 、 \mathrm{Rc} 、 \mathrm{Re} 、 \mathrm{Rf} 、 \mathrm{Rg} 1)$ 含量与月降水量 $(\mathbf{A})$ 和月平均气温 $(\mathbf{B})$ 的圥余分析。 $\mathrm{AP} 1-\mathrm{AP} 12,1-12$ 月月 降水量; TM1-TM12, 1-12月月平均气温。

Fig. 3 Redundancy analysis of average monthly precipitation (A), average monthly temperature (B) and ginsenosides (Rb1, Rb2, $\mathrm{Rc}, \mathrm{Re}, \mathrm{Rf}, \mathrm{Rg} 1$ ) content in Panax ginseng. AP1-AP12, average monthly precipitation of Jan. to Nov.; TM1-TM12, average monthly temperature of Jan. to Nov. 
人参皇苷含量正相关。6-9月为人参的主要生长期, 说明人参主要生长期中高温抑制人参皇苷的积累, 但其他时间较高的温度则有利于人参皇苷的积累。 这与上文中较温暖环境适合㿝苷积累、高温是人参 皇苷积累的主要限制因素的结果相符。

\section{4 土壤因子与皇苷含量相关性分析}

为分析土壤中营养元素与人参皇苷含量关系, 采用圥余分析对 5 种人参特征皇苷与土壤营养元素 进行相关性分析(图4)。前两个排序轴对皇苷含量与 环境因子的解释度为 $81.7 \%$, 说明在人参㿝苷含量 与土壤因子间存在显著的相关关系。并且前两个排 序轴解释了不同产地间人参皇苷含量间变异的 $61.9 \%$ 。排序轴 1 与全氮、 $\mathrm{Fe}$ 含量显著正相关 (相关系 数分别为 $0.583 、 0.444)$, 与 $\mathrm{K}$ 含量显著负相关(相关 系数为 -0.251 ); 排序轴 2 与全氮含量、 $\mathrm{pH}$ 值显著负 相关 (相关系数分别为 $-0.176 、-0.128)$, 与有机质、 $\mathrm{Mn}$ 含量显著正相关(相关系数分别为 $0.270 、 0.218$ )。 图4显示, 人参㿝苷 $\mathrm{Rg} 1 、 \mathrm{Rb} 1$ 含量与全氮、 $\mathrm{Fe}$ 含量 负相关，与 $\mathrm{K}$ 含量正相关; 人参㿝苷 $\mathrm{Re}$ 含量与全氮、 $\mathrm{Fe} 、 \mathrm{Mn} 、 \mathrm{P}$ 含量正相关，与 $\mathrm{K}$ 含量负相关; 人参皇苷 $\mathrm{Rf} 、 \mathrm{Rc} 、 \mathrm{Rb} 2$ 含量与有机质、 $\mathrm{Mn} 、 \mathrm{Zn} 、 \mathrm{P}$ 含量正相 关, 与 $\mathrm{pH}$ 值负相关。

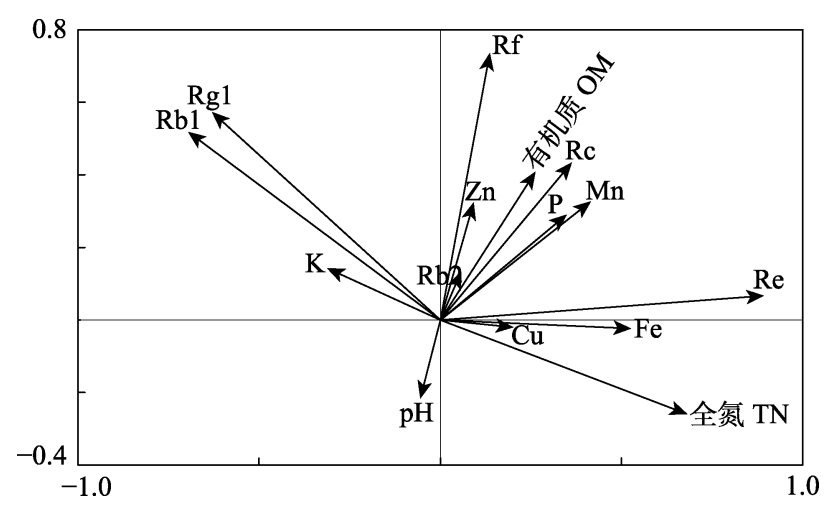

图4 人参皇苷 $(R b 1 、 R b 2 、 R c 、 R e 、 R f 、 R g 1)$ 含量与土壤 因子咒余分析。

Fig. 4 Redundancy analysis of soil factors and ginsenosides (Rb1, Rb2, Rc, Re, Rf, Rg1) content in Panax ginseng. OM, organic matter; $\mathrm{TN}$, total nitrogen.

\section{3 讨论}

药用植物的品质除决定于物种的遗传特性, 生 态环境对物种亦起重要作用(曾燕等, 2012)。本研究 对药用植物有效成分的空间变异进行分析, 并研究 其与土壤、气候因子的相关性。文中将化学成分分 析和生态因子分析相结合, 分析了 3 个不同产地的
28 批人参样品中 9 种活性皇苷与 9 个气候因子、 16 批 人参样品中 9 个土壤中营养元素的相关性, 确定了 对人参皇苷积累影响较大的气候因子与土壤因子。

气候因子中, 影响人参㿝苷含量的主要气候因 子是水分、热量和光照, 并且水分因子对人参生长 的影响最大。年降水量与人参㿝苷含量均为正相关, 9月降水量与人参皇苷含量负相关, 所以人参种植 中应注意控制 9 月的水分因子; 6-9月月平均气温与 人参㿝苷含量均为负相关, 其余月份月平均气温与 人参㿝苷含量均为正相关, 年平均气温与人参皇苷 含量为正相关; 年日照时间与人参㿝苷含量均为负 相关。上述分析结果说明温暖、潮湿、阴凉的环境 适合㿝苷积累, 㿝苷具有不耐高温的特性。这与沈 亮等(2015)在人参种植体系研究中, 得出人参最适 湿度30\%-60\%, 最适温度 $10-30{ }^{\circ} \mathrm{C}$, 喜漫射光和散 射光, 忌强光直射的研究结论基本一致。

土壤因子中全氮、 $\mathrm{Fe} 、 \mathrm{~K}$ 、有机质、 $\mathrm{Mn} 、 \mathrm{P}$ 、 $\mathrm{Zn}$ 含量、 $\mathrm{pH}$ 值对人参㿝苷含量的影响较大。植物主 要从土壤中吸收水分和营养物质, 土壤中无机元素 的含量反映了土壤的养分, 均衡的土壤养分对人参 品质至关重要。程海涛等(2011)指出, 土壤 $\mathrm{pH}$ 值是 土壤各种化学性质的反应, 对土壤肥力、有机质的 合成与分解, 各种营养物质的转化和释放都有影响, $\mathrm{N} 、 \mathrm{P} 、 \mathrm{~K}$ 是人参栽培中必不可少的元素，与赵英等 (2001)得出微量元素与P、K配合施用对人参单支重 增产效果更为显著的结论基本一致。

中药材与生态环境的相关性研究, 阐明了生态 环境因素对药材品质形成的影响(段金倣等, 2009)。 上述分析结果显示少数生态因子对皇苷含量影响显 著, 多数与皇苷含量相关性较小, 但是药材品质是 多重生态因子作用的结果, 各生态因子间相互影响, 共同决定药用植物的品质，所以，也不能忽略相关 性较小的生态因子对药材品质形成的影响。

本文通过研究人参皇苷含量的空间变异及其 与生态环境因素间的相关性明确了影响人参皇苷含 量空间变异的主要生态因子, 但是仅用化学成分含 量评价人参品质具有一定的局限性, 在后续工作中, 可以对人参品质进行深入的研究, 从药效入手, 以 效(药效, 效价) 定质(品质), 以质论环(生态环境), 构建“效-质-环”的研究体系(滒丹等, 2012)。

基金项目 国家自然科学基金(81473304)和国家科 技支撑计划(2015BAI05B01)。 


\section{参考文献}

Chen SL, Suo FM, Han JP, Xie CX, Yao H, Li XW, Li Y, Wei JH (2007). Analysis on ecological suitability and regionalization of traditional Chinese medicinal materials. Chinese Traditional and Herbal Drugs, 38, 481-487. (in Chinese with English abstract) [陈士林, 索风梅, 韩建平, 谢 彩香, 姚辉, 李西文, 李漟, 魏建和 (2007). 中国药材 生态适宜性分析及生产区划. 中草药, 38, 481-487.]

Cheng HT, Zhang YY, Zhang LX, Xu YH (2011). The progress of research on the relation of soil environment and Panax ginseng. Journal of Chinese Medicinal Materials, 34, 313318. (in Chinese) [程海涛, 张亚玉, 张连学, 许永华 (2011). 土壤环境与人参生长关系的研究进展. 中药材, 34, 313-318.]

Dong JZ, Wang SH, Zhu LY, Wang Y (2012). Analysis on the main active components of Lycium barbarum fruits and related environmental factors. Journal of Medicinal Plants Research, 6, 2276-2283.

Duan JA, Zhou RH, Su SL, Ding AW, Wu QN (2009). Present situation and prospects of development of resources science of Chinese medicinal materials in China. Journal of Natural Resources, 24, 378-387. (in Chinese with English abstract) [段金倣，周荣汉，宿树兰，丁安伟，吴启楠 (2009). 我国中药资源科学发展现状及展望. 自然资源 学报, 24, 378-387.]

Huang LF, Xie CX, Duan BZ, Chen SL (2010). Mapping the potential distribution of high artemisinin-yielding Artemisia annua L. (Qinghao) in China with a geographic information system. Chinese Medicine, 5, 18. doi: 10.1186/1749-8546-5-18.

Jia GL, Huang LF, Suo FM, Song JY, Xie CX, Sun J (2012). Correlation between ginsenoside contents in Panax ginseng roots and ecological factors, and ecological division of ginseng plantation in China. Chinese Journal of Plant Ecology, 36, 302-312. (in Chinese with English abstract) [贾光林, 黄林芳, 索凤梅, 宋经元, 谢彩香, 孙娟 (2012). 人参药材中人参皇苷与生态因子的相关性及人 参生态区划. 植物生态学报, 36, 302-312.]

Li H, Xu L, Wen MJ, Zeng FL, Xie CX, Kang TG (2015). Study on the content measurement and quality evaluation of ginsenoside constituent from different habitats with UPLC analysis method. China Journal of Traditional Chinese Medicine and Pharmacy, 30, 1963-1968. (in Chinese with English abstract) [李慧, 许亮, 温美佳, 曾 凡琳, 谢彩香, 康廷国 (2015). 不同产地人参皇苷成分 含量UPLC法测定及质量评价. 中国中医药杂志, 30 , 1963-1968.]

Lin MY, Liu HY, Liu JP, Lu D, Li PY (2011). Progress of chemical research of ginsenosides. Ginseng Research, 23(4), 43-46. (in Chinese with English abstract) [林美妤, 刘海宇, 刘金平, 卢丹, 李平亚 (2011). 人参皇苷的化
学研究进展. 人参研究, 23(4), 43-46.]

Meng XC, Yu DM, Sun H, Yang GH, Wang XJ (2010). Study on relationship between active ingredients in stem of Acanthopanax senticosus and ecological factors by partial least-squares regression. Research and Practice on Chinese Medicines, 24, 17-21. (in Chinese with English abstract) [孟祥才, 于冬梅, 孙晖, 杨国辉, 王喜军 (2010). 偏最小二乘回归分析刺五加茎活性成分与生态因子相 关性. 现代中药研究与实践, 24, 17-21.]

Shen L, Xu J, Dong LL, Li XW, Chen SL (2015). Cropping system and research strategies in Panax ginseng. China Journal of Chinese Meteria Medica, 40, 3367-3374. (in Chinese with English abstract) [沈亮, 徐江, 董林林, 李 西文, 陈士林 (2015). 人参栽培种植体系及其研究策 略. 中国中药杂志, 40, 3367-3374.]

Tang SH, Yang HJ, Huang LQ (2010). Discuss on effect of physical environmental factors on nature of Chinese meteria medica. China Journal of Chinese Meteria Medica, 35, 126-128. (in Chinese with English abstract) [唐仕欢, 杨 洪军, 黄璐琪 (2010). 论自然环境因子变化对中药药性 形成的影响. 中国中药杂志, 35, 126-128.]

Tao SH, Wu FY (2003). Effect of ecological environment on active constituents of medicinal plants. Natural Product Research and Development, 15, 174-178. (in Chinese with English abstract) [陶曙红, 吴凤锷 (2003). 生态环境对 药用植物有效成分的影响. 天然产物研究与开发, 15 , 174-178.]

Xie CX, Suo FM, Jia GL, Song JY, Huang LF, Chen SL (2011). Correlation between ecological factors and ginsenosides. Acta Ecologica Sinica, 31, 7551-7546. (in Chinese with English abstract) [谢彩香, 索凤梅, 贾光林, 宋经 元, 黄林芳, 陈士林 (2011). 人参皇苷与生态因子的相 关性. 生态学报, 31, 7551-7564.]

Xie CX, Suo FM, Zhou YQ, Wang LZ, Xiang L, Dong L, Jia GL, Sun CZ, Chen SL (2011). Qualitative study on ecological suitability of Chinese herbal medicine based on GIS. China Journal of Chinese Meteria Medica, 36, 379-382. (in Chinese with English abstract) [谢彩香, 索凤梅, 周应 群, 王丽芝, 向丽, 董梁, 贾光林, 孙成忠, 陈士林 (2011). 基于地理信息系统的中药材生态适宜性定量化 研究. 中国中药杂志, 36, 379-382.]

Xu J, Jia L, Zhao YQ (2011). Constituents of ginseng and quality evaluation of ginseng products. Drug Evaluation Research, 34, 199-202. (in Chinese with English abstract) [徐静, 贾力, 赵余庆 (2011). 人参的化学成分与人参 产品的质量评价. 药物评价研究, 34, 199-202.]

Xu XY, Zheng YM, Fu SQ, Zhao Y, Li J, Wang LL (2011). Determination of twelve ginsenosides in Panax ginseng by HPLC. China Journal of Chinese Meteria Medica, 36, 1463-1465. (in Chinese with English abstract) [胥秀英, 郑一敏, 傅善权, 赵颖, 李杰, 王琳琳 (2011). HPLC同 时测定人参药材中 12 种人参皇苷的含量. 中国中药杂 
志, 36, 1463-1465.]

Yan D, Wang JB, Li JX, Ma LN, Xiao XH (2012). Strategy for research on quality identification and ecological environment-related of Dao-di herb. China Journal of Chinese Meteria Medica, 37, 2672-2676. (in Chinese with English abstract) [駱丹, 王伽伯, 李俊贤, 马丽娜, 肖小 河 (2012). 论道地药材品质辨识及其与生态环境的相 关性研究策略. 中国中药杂志, 37, 2672-2676.]

Yang ST, Li JH, Zhao YP, Chen BL, Fu CX (2011). Harpagoside variation is positively correlated with temperature in Scrophularia ningpoensis Hemsl. Agricultural and Food Chemistry, 59, 1612-1621.

Zeng Y, Guo LP, Yang G, Chen BD, Wang JY, Huang LQ (2012). Effect of environmental ecological factors on saponins of medicinal plant. Chinese Journal of Experimental Traditional Medical Formulae, 18, 313-318. (in Chinese with English abstract) [曾燕, 郭兰萍, 杨光, 陈 保东, 王继勇, 黄璐琦 (2012). 环境生态因子对药用植 物㿝苷成分的影响. 中药材, 18, 313-318.]

Zhang CY, Dong L, Chen SL, Xie CX, Chang DL (2010). UPLC fingerprint for quality assessment of ginsenosides of Ginseng radix et Rhizoma. Acta Pharmaceutica Sinica, 45, 1296-1300. (in Chinese with English abstract) [张翠 英, 董梁, 陈士林, 谢彩香, 常断玲 (2010). 人参药材 㿝苷类成分UPLC特征图谱的质量评价方法. 药学学报,
45, 1296-1300.]

Zhang JZ, Zhang WJ, Mu Q, Luo JP (2011). Research advance on pharmacological activity and content accumulation of chemical component from Ginseng. Journal of Anhui Agricultural Sciences, 39, 12158-12160. (in Chinese with English abstract) [张翼输, 张文驹, 穆青, 罗建平 (2011). 人参化学成分的药理活性及其含量积累的研究 进展. 安徽农业科学, 39, 12158-12160.]

Zhao Y, Wang XQ, Zheng YN, Ren YY, Liu GY (2001). Effect of inorganic fertilizers on yields properties of ginseng. Journal of Jilin Agricultural University, 23(4), 56-59. (in Chinese with English abstract) [赵英, 王秀全, 郑毅男, 任跃英, 刘桂艳 (2001). 施用化肥对人参产量性状的影 响. 吉林农业大学学报, 23(4), 56-59.]

Zhu JJ, Wang ZM, Kuang YH, Zhang QW, Gao QP, Ma N (2008). A quantitative method using one marker for simultaneous assay of ginsenosides in Panax ginseng and P. notoginseng. Acta Pharmaceutica Sinica, 43, 12111216. (in Chinese with English abstract) [ 朱晶晶, 王智民, 匡艳辉, 张启伟, 高其品, 马妮 (2008). 一测多评法同 步测定人参和三七药材中多种人参㿝苷的含量. 药学 学报, 43, 1211-1216.]

责任编委: 王根轩 责任编辑: 李 敏

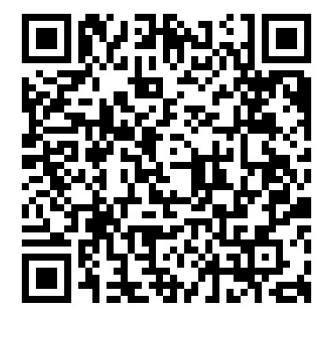

www.plant-ecology.com 